\title{
Nucleation and growth of helium bubbles in aluminum between 20 and $900 \mathrm{~K}$
}

\author{
Rajainmäki, H.; Linderoth, Søren; Hansen, H. E.; Nieminen, R. M.; Bentzon, M. D.
}

Published in:

Physical Review B

Link to article, DOI:

10.1103/PhysRevB.38.1087

Publication date:

1988

Document Version

Publisher's PDF, also known as Version of record

Link back to DTU Orbit

Citation (APA):

Rajainmäki, H., Linderoth, S., Hansen, H. E., Nieminen, R. M., \& Bentzon, M. D. (1988). Nucleation and growth of helium bubbles in aluminum between 20 and 900 K. Physical Review B, 38(2), 1087-1094.

https://doi.org/10.1103/PhysRevB.38.1087

\section{General rights}

Copyright and moral rights for the publications made accessible in the public portal are retained by the authors and/or other copyright owners and it is a condition of accessing publications that users recognise and abide by the legal requirements associated with these rights.

- Users may download and print one copy of any publication from the public portal for the purpose of private study or research.

- You may not further distribute the material or use it for any profit-making activity or commercial gain

- You may freely distribute the URL identifying the publication in the public portal 


\title{
Nucleation and growth of helium bubbles in aluminum between 20 and $900 \mathrm{~K}$
}

\author{
H. Rajainmäki \\ Department of Physics, University of Jyväskylä, SF-40100 Jyväskylä, Finland
}

S. Linderoth and H. E. Hansen*

Laboratory of Applied Physics II, Technical University of Denmark, DK-2800 Lyngby, Denmark

\author{
R. M. Nieminen ${ }^{\dagger}$ \\ Department of Physics, University of Jyväskylä, SF-40100 Jyväskylä, Finland
}

M. D. Bentzon

Laboratory of Applied Physics I, Technical University of Denmark, DK-2800 Lyngby, Denmark

(Received 22 September 1987; revised manuscript received 22 February 1988)

\begin{abstract}
The nucleation and growth of helium bubbles have been monitored in ${ }^{3} \mathrm{He}$-implanted aluminum by the positron-lifetime technique and transmission electron microscopy. Helium atoms are mobile during the implantation and are subsequently trapped by vacancies. The annealing stages I and III are observed at 40 and $200 \mathrm{~K}$, respectively, in agreement with previous positron annihilation and electrical-resistivity data. The low implantation temperature of $20 \mathrm{~K}$ hinders bubble formation during implantation, but during isochronal annealing, around $425 \mathrm{~K}$, helium starts to nucleate into bubbles via substitutional migration of the He-vacancy pairs. The migration energy for a $\mathrm{He}$ vacancy pair is estimated to be $1.3 \pm 0.1 \mathrm{eV}$. Above $600 \mathrm{~K}$ the He bubbles grow through condensation of thermally produced vacancies, as well as bubble migration and coalescence. The created helium bubbles are extremely stable and survive the final $900-\mathrm{K}$ annealing. The helium density, as a function of annealing temperature, was determined from the positron-lifetime data employing recent theoretical calculations. Also, the bubble size was estimated. The presence of helium bubbles was verified with transmission electron microscopy and they were found to be polygon shaped. The fraction of positrons annihilating in the helium bubbles was found to decrease when lowering the temperature below room temperature, in accord with results on empty voids in aluminum.
\end{abstract}

\section{INTRODUCTION}

Helium in metals is technologically problematic, mainly due to its role in the swelling of fission-reactor materials. ${ }^{1}$ Helium, being a closed-shell atom, interacts repulsively with metal atoms and, hence, has a very low solubility in metals. Thus it is effectively trapped at any open-volume defects and has a tendency to precipitate and form bubbles at elevated temperatures. Recently, both experimental and theoretical interest in studying the behavior of rare-gas atoms in metals has increased due to the fact that the introduction rate of $\mathrm{He}$ into the first-wall materials in future fusion reactors will be very high. ${ }^{2}$ However, the fundamental understanding of the microscopic behavior of helium in metals is still incomplete, mainly due to the lack of detailed experimental data.

Several techniques have been developed in order to characterize the behavior of $\mathrm{He}$ in metals., ${ }^{3,4}$ Thermalhelium-desorption spectroscopy has been utilized to study the migration of substitutional $\mathrm{He}$ in metals. ${ }^{5}$ The interstitial migration energy of $\mathrm{He}$ has not been accurately determined for $\mathrm{Al}$, but is known to be very low ( $\mathrm{He}$ is mobile below $100 \mathrm{~K}){ }^{4}$ The most direct way to study He bubbles is by transmission electron microscopy (TEM). This technique is, however, limited to large bubbles, since the detection limit of TEM is typically $>1 \mathrm{~nm}$. Several other techniques, such as electron-energy-loss spectroscopy (EELS), lattice-parameter change ( $\Delta a / a)$, small-angle neutron (SANS) and $x$-ray scattering (SAXS), and vacuum-ultraviolet-absorption spectroscopy (VUVAS), have been utilized in order to reveal information on $\mathrm{He}$ in bubbles in metals. The density determinations for $\mathrm{He}$ in bubbles in metals have recently been reviewed by Donnelly. 6

A technique, which is particularly sensitive to small submicroscopic vacancy-type clusters, such as $\mathrm{He}$ bubbles, is the positron-lifetime technique, ${ }^{7}$ which has been widely applied in studies of vacancy-type defects and their interactions in metals. It is selectively sensitive to open-volume defects which are effective traps for positrons. The lifetime associated with the defect depends on the size of the cavity, being 245 ps in a monovacancy in Al and increasing up to about 500 ps (Ref. 8) in a large void (more than 30 vacancies). Furthermore, the decoration of the cavities by impurities changes the associated lifetime, ${ }^{9}$ which enables positron studies of impuritydefect interactions. The positron-lifetime technique is sensitive to vacancies in the concentration range above $0.1 \mathrm{ppm}$, and above $100 \mathrm{ppm}$ practically all positrons become trapped by vacancies.

Helium injected into Al was first studied using the positron-lifetime technique by Snead et al., ${ }^{10}$ who ob- 
served the formation of small helium-vacancy precipitates after room-temperature implantation. As a result of the small $\mathrm{He}$ concentration $(0.6 \mathrm{ppm})$ the disappearance of the bubbles or He-vacancy clusters was observed around $825 \mathrm{~K}$, presumably because the bubble concentration went below the detection limit for the positronlifetime technique. Hansen et al. ${ }^{11}$ implanted $\mathrm{He}$ into $\mathrm{Al}$ below $475 \mathrm{~K}$ to a dose of $1000 \mathrm{ppm} \mathrm{He}$, and they observed significant helium-bubble growth during an isochronal annealing. The bubbles created were extremely stable and survived annealing up to the Al melting temperature. Jensen et al. ${ }^{12}$ have studied $\mathrm{He}$ bubbles introduced into $\mathrm{Al}$ by irradiation with $600-\mathrm{MeV}$ protons at various temperatures $(400-700 \mathrm{~K})$ and with different doses (30-100 ppm He). The samples were investigated with the positron-lifetime technique and TEM in order to correlate the positron lifetime and the helium density in the bubbles.

Jensen and Nieminen ${ }^{13}$ have recently done detailed molecular-dynamics simulations and positron-state calculations in order to obtain a relation between the helium density and the corresponding positron lifetime in the bubbles. They also calculated the positron lifetime versus the size of the vacancy clusters for different heliumvacancy number ratios $([\mathrm{He}] /[V])$ in the clusters. These results are useful for unambiguous gas-density determinations.

In the present experiment $26.5-\mathrm{MeV}{ }^{3} \mathrm{He}$ ions were implanted into aluminum at $20 \mathrm{~K}$ to a dose of $1000 \mathrm{ppm} \mathrm{He}$. Isochronal annealing experiments, followed by positronlifetime measurements, were performed between 20 and $900 \mathrm{~K}$. The nucleation of submicroscopic bubbles is clearly detected in the present experiment. The He density in the bubbles and the bubble radii are estimated from the positron-lifetime results, based on the recent theoretical calculations. The bubble-growth mechanisms are discussed in the light of the results.

\section{EXPERIMENTAL DETAILS}

Well-annealed and carefully electropolished Al single crystals of $99.9999 \%$ purity were implanted with 26.5$\mathrm{MeV}{ }^{3} \mathrm{He}$ ions ejected from the MC-20 cyclotron at the University of Jyväskylä. The specimens (thickness 385 $\mu \mathrm{m}$, diameter $12 \mathrm{~mm}$ ) were mounted in a sample-sourcesample sandwich geometry in the irradiation cryostat, where they could be kept below $20 \mathrm{~K}$ during the implantation and the subsequent positron-lifetime measurements. The details of the experimental setup are described elsewhere. ${ }^{14,15}$

The use of ${ }^{3} \mathrm{He}$ instead of ${ }^{4} \mathrm{He}$ is due to experimental reasons only; the maximum kinetic energy that can be achieved for ${ }^{4} \mathrm{He}$ in the cyclotron is $20 \mathrm{MeV}$, which is not sufficient to penetrate the minimum sample thickness needed for the positron measurements. The isotope effect is not expected to play any significant role otherwise. The thickness of the samples was chosen in such a way that about $99 \%$ of the positrons were made to stop in the specimens. The range of the $26.5-\mathrm{MeV}^{3} \mathrm{He}$ ions $(375 \mu \mathrm{m})$ was extracted from the Bethe formula ${ }^{16}$ and found to be in good agreement with the available experimental data. ${ }^{17}$
The straggling of the ions in Al is about $5 \mu \mathrm{m}$. A stack of $5-\mu \mathrm{m}$ Al foils ( $0-8$ pieces) was used as a beam-energy degrader in order to obtain a more homogeneous implant distribution. The helium concentrations given in this paper are the mean values in the implantation volume.

The implantation profile of positrons from the radioactive source is nearly exponential. The expression of Linderoth et al. ${ }^{18}$ gives a mass absorption coefficient of 45 $\mathrm{cm}^{2} / \mathrm{g}$ for positrons from ${ }^{22} \mathrm{Na}$ implanted into $\mathrm{Al}$. By applying this value, we can estimate that about $50 \%$ of the positrons stop in the He-implanted region and $40 \%$ annihilate in the damaged, He-free region. About $10 \%$ of the positrons annihilate in the defect-free region between the positron source and the damaged area. The results above 300-K annealing, however, suggest that the fraction of the positrons annihilating in the He-implanted region is higher, about $70 \%$, which may be explained by a somewhat larger $\mathrm{He}$ range or smaller specimen thickness than estimated.

The positron-lifetime measurements for the annealings between 20 and $300 \mathrm{~K}$ were performed at $15 \mathrm{~K}$ with a fast-coincidence-time spectrometer ${ }^{19}$ having a time resolution of 300 ps [full width at half maximum (FWHM)] as deduced by the RESOLUTION program ${ }^{20}$ from measurements on a defect-free sample set. From the same spectra the lifetime and the intensity associated with the annihilation in the positron source were found to be 400 ps and $5 \%$, respectively. A second source component, associated with annihilation in the source-confining metal foil, was estimated to be $13 \%$ of 170 ps utilizing the empirical determinations by Linderoth et al. ${ }^{18}$ The spectra for annealings above $300 \mathrm{~K}$ were acquired at $80 \mathrm{~K}$ by a fastfast-coincidence-time spectrometer. The time resolution ( $250 \mathrm{ps}, \mathrm{FWHM}$ ) and the source component (163 ps, 8\% and $450 \mathrm{ps}, 4 \%$ ) were deduced in a similar manner as described above. The isochronal annealing was performed with $12.5-\mathrm{K}$ steps up to $300-\mathrm{K}$ annealing and continued with $25-\mathrm{K}$ steps. The annealing rate was kept constant, $50 \mathrm{~K} / \mathrm{h}$, during the whole annealing treatment. Only one repacking, at $300 \mathrm{~K}$, was required before annealing up to $600 \mathrm{~K}$. Above $600 \mathrm{~K}$ the source had to be removed during the annealings and consequently the sample set was repacked after each annealing. All the annealings were carried out in vacuum.

After each annealing one to four positron-lifetime spectra were acquired, each spectrum containing $(0.8-1.0) \times 10^{6}$ counts. The lifetime spectra were analyzed with the POSITRONFIT program. ${ }^{20}$ Two-exponential fittings were applied for all spectra, but for annealings below $300 \mathrm{~K}$ the statistical scatter of the deduced parameters led us to employ the more stable mean lifetime,

$$
\bar{\tau}=\sum_{i=1}^{N} I_{i} \tau_{i},
$$

where $I_{i}$ and $\tau_{i}$ are the individual intensities and lifetimes of the $N$ components (usually, $N=2$ ). The mean lifetime is less informative than the two-component fit, but gives enough information to give a clear picture of the annealing behavior below $300 \mathrm{~K}$.

One of the samples was studied by TEM after the 900K annealing employing the Philips EM 430 transmission 
electron microscope with an electron energy of $300 \mathrm{keV}$ and a magnification of $5 \times 10^{5}$. A disk $3 \mathrm{~mm}$ in diameter was drilled from the beam spot. The disk was polished, first mechanically from the positron-source side and then electrolytically from the other side, until the sample was suitable for TEM investigations.

\section{RESULTS AND DISCUSSION}

\section{A. As-implanted aluminum}

During the implantation the dose dependence of the positron lifetime was also measured and the results are seen in Fig. 1. From the positron-lifetime measurement at $15 \mathrm{~K}$ on the well-annealed specimens the positron lifetime of $156 \mathrm{ps}$ is deduced, which is consistent with previous measurements on nominally defect-free specimens. ${ }^{15}$ The $\mathrm{He}$ dose was made to increase exponentially from 1 to 1000 at ppm. The mean positron lifetime, $\bar{\tau}$, measured after each implantation increased due to the increased concentration of vacancies.

The 1-ppm implantation increased the mean lifetime to 193 ps. Because nine irradiations were performed on each side to obtain an implanted region of about $40 \mu \mathrm{m}$, the 1 -ppm dose corresponds to about $2 \times 10^{14}$ impinging ions per $\mathrm{cm}^{2}$. The subsequent implantation to an accumulated dose of $10 \mathrm{ppm}$ increased the mean lifetime by only $1 \mathrm{ps,}$, presumably due to nearly saturated positron trapping at vacancies and He-vacancy pairs in the implanted region already after the 1-ppm implantation. The stronger increase after $100 \mathrm{ppm}$ may be assigned to increased positron trapping in the irradiated but unimplanted region.

Each $\mathrm{He}$ ion produces several Frenkel pairs in its stopping region. ${ }^{2,21}$ At $20 \mathrm{~K}$ most of the Frenkel pairs avoid recombination, because neither interstitials nor vacancies are mobile. Since the vacancy concentration has far ex-

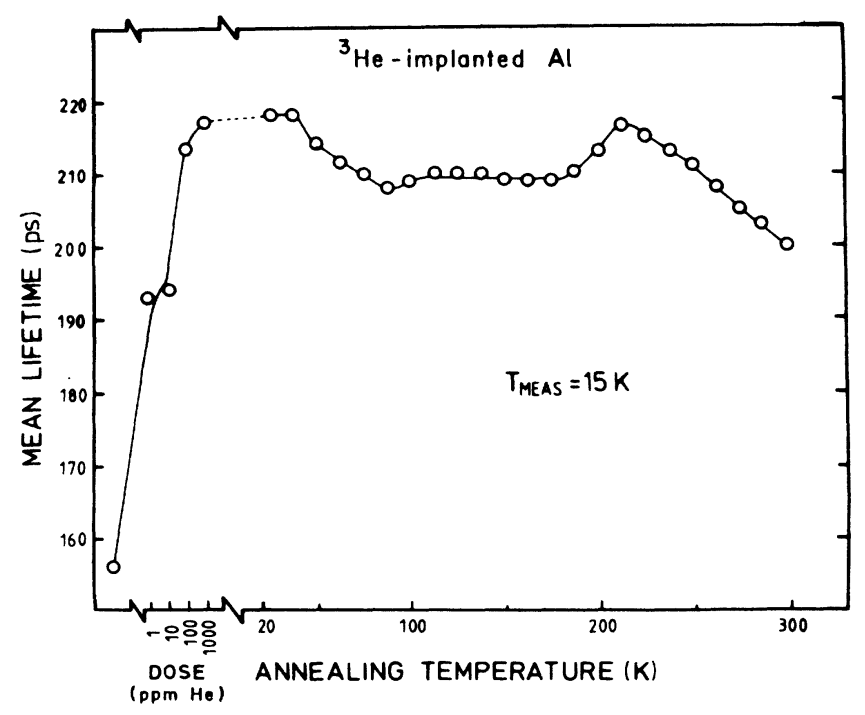

FIG. 1. Mean positron lifetime at $15 \mathrm{~K}$ in $26.5-\mathrm{MeV}{ }^{3} \mathrm{He}-$ implanted $\mathrm{Al}$ vs the implantation dose and isochronal annealing temperature. The line is drawn only to guide the eye. ceeded $100 \mathrm{ppm}$ after the implantation of $100 \mathrm{ppm} \mathrm{He}$, the positron trapping is saturated in the implanted region. During the $1000-\mathrm{ppm}$ implantation the defectproduction rate also becomes saturated due to spontaneous Frenkel-pair annihilation. The maximum Frenkelpair concentration in fcc metals is typically 5000-10000 ppm. ${ }^{22}$ Thus we estimate that the He-vacancy concentration ratio in the implantation region is approximately 0.2 after the 1000-ppm implantation.

\section{B. Annealing from 20 to $300 \mathrm{~K}$}

Figure 1 shows the mean positron lifetime measured at $15 \mathrm{~K}$ during the annealing up to $300 \mathrm{~K}$. The mean lifetime decreases from 218 ps at $35 \mathrm{~K}$ to $208 \mathrm{ps}$ at $85 \mathrm{~K}$. Some increase is observed between 85 and $120 \mathrm{~K}$ and the mean lifetime remains constant up to $200 \mathrm{~K}$. At $200 \mathrm{~K}$ the mean lifetime starts to increase, being 216 ps at 220 K. Then it starts to decrease, which continues up to 300 $\mathrm{K}$. After the $300-\mathrm{K}$ annealing the mean lifetime is 200 ps, which indicates that an appreciable amount of positron traps are still present.

Three different reactions are seen in the temperature interval $25-130 \mathrm{~K}$. The similar behavior in the mean lifetime during the annealing is also seen after proton implantation ${ }^{15}$ and proton irradiation ${ }^{23}$ in aluminum. These results clearly indicate that the changes in the mean lifetime are due to the intrinsic properties of the host crystal. The rapid decrease between 25 and $50 \mathrm{~K}$ is assigned to free migration of interstitials (stage I) and their recombination with vacancies, in agreement with recent positron-lifetime ${ }^{15}$ and resistivity ${ }^{24}$ results. The behavior between 50 and $130 \mathrm{~K}$ is due to interstitial clustering and rearrangement and is discussed in detail elsewhere. ${ }^{25}$

At $200 \mathrm{~K}$ a significant increase in the mean lifetime is seen due to the migration and clustering of vacancies (stage III). A very high concentration of vacancies, together with the possible role of immobile He-vacancy complexes as nucleation centers, makes clustering favorable and the cavity size increases, leading to an increased positron lifetime. ${ }^{26}$ The vacancy clusters are not sufficiently large to remain stable, but dissociate at elevated annealing temperatures above $220 \mathrm{~K}$.

The changes of the mean lifetime between 20 and 300 $\mathrm{K}$ can all be assigned to vacancies and interstitials. No sign of the He migration and trapping by vacancies is observed in that temperature range. The positron lifetime in the $\mathrm{He}$ decorated vacancy is about 40 ps shorter than in a clean vacancy, ${ }^{9}$ and the He trapping during the annealing would therefore be observed by changes of the mean lifetime. We therefore conclude that $\mathrm{He}$ is mobile during the implantation. Interstitial helium has been found to be mobile at around $100 \mathrm{~K} .^{4}$ The lower temperature for $\mathrm{He}$ mobility, in the present experiment, may be due to epithermal mobility or nonthermal processes (i.e., the nearly saturated vacancy concentration can facilitate correlated $\mathrm{He}$ migration to the vacancies). Since there are about 5 times more empty vacancies than $\mathrm{He}$-vacancy pairs, the probability for multiple He occupation of vacancies during the implantation is very low. Because $\mathrm{He}$-vacancy pairs are not mobile at this temperature, ${ }^{5}$ no He bubbles were formed during the implantation. 


\section{Annealing above $300 \mathrm{~K}$}

Figure 2 shows the positron-lifetime parameters, measured at $77 \mathrm{~K}$, during the isochronal annealing from 300 to $900 \mathrm{~K}$. The high stability and the good time resolution of the spectrometer allowed us to reliably resolve two exponentials from the lifetime spectra after the $300-\mathrm{K}$ annealing. At $300 \mathrm{~K}$ the longer-lifetime component, $\tau_{2}$, is about 250 ps with the intensity $I_{2} \simeq 60 \%$. From 300 to $450 \mathrm{~K}$ the longer lifetime decreases down to about $205 \mathrm{ps}$. Between 450 and $500 \mathrm{~K}, \tau_{2}$ increases rapidly and $I_{2}$ starts to decrease. Also, $\tau_{1}$ increases slightly, in accord with the standard trapping model. ${ }^{7}$ Between 500 and $650 \mathrm{~K}$ the increase of $\tau_{2}$ levels off, becoming again more rapid above $650 \mathrm{~K}$. The lifetime $\tau_{2}$ after the final $900-\mathrm{K}$ annealing is $469 \mathrm{ps}$, which is close to the lifetime in a large void. The decrease of $I_{2}$ continues slowly above $500 \mathrm{~K}$ up to $900 \mathrm{~K}$.

To be able to give an estimate of the $[\mathrm{He}] /[\boldsymbol{V}]$ ratio, we shall look at the reactions that may have an influence on the ratio during the annealing. During the implantation at $20 \mathrm{~K}$ mainly monovacancies and interstitials are created. The migration energy of $\mathrm{He}$ in $\mathrm{Al}$ is very low, causing some of the vacancies (about 20\%) to become decorated by $\mathrm{He}$ atoms during the implantation. The

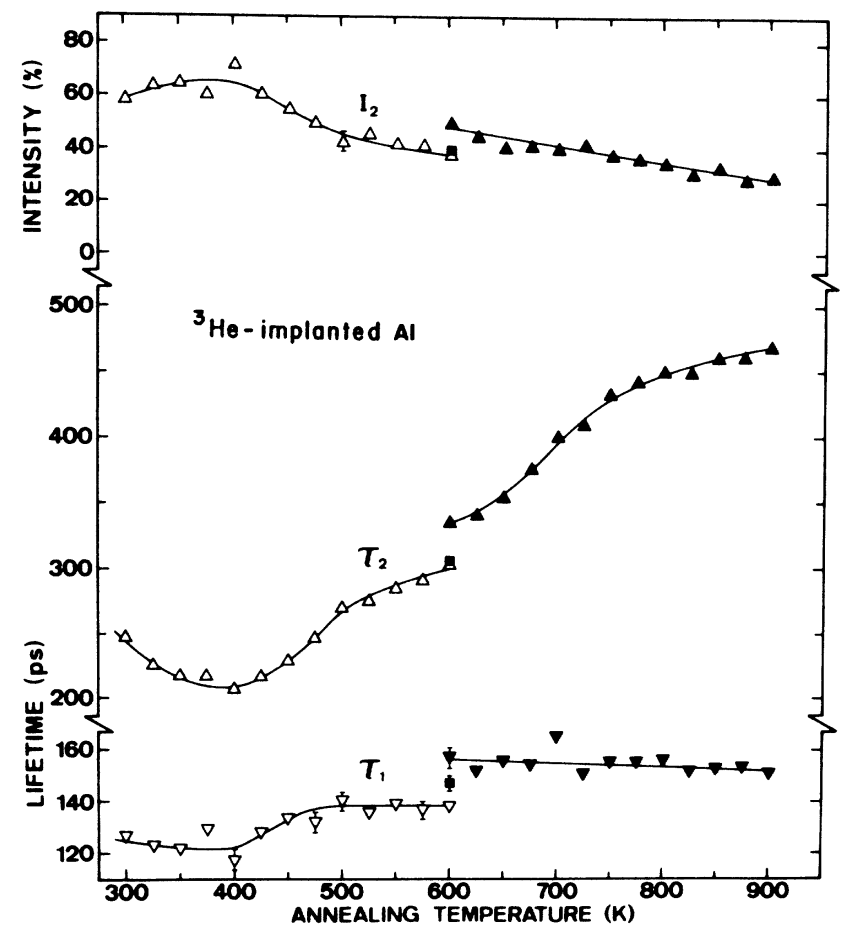

FIG. 2. Positron-lifetime parameters at $77 \mathrm{~K}$ in $26.5-\mathrm{MeV}$ ${ }^{3} \mathrm{He}$-implanted aluminum vs the annealing temperature. Open triangles have been measured with the same sample-sourcesample packing. Solid triangles have been measured with the sample sandwich repacked before each measurement. The step at $600 \mathrm{~K}$ is only due to improper packing (the source was slightly off the center of the beam spot) before the $600-\mathrm{K}$ annealing. This is demonstrated with the solid square at $600 \mathrm{~K}$ where the source was placed off center and the values of the parameters before repacking are reproduced. The lines are drawn only to guide the eye. probability of having two $\mathrm{He}$ atoms in the same vacancy during the implantation is low due to the large amount of available empty vacancies. In stage $I$, interstitial atoms become mobile and annihilate with vacancies (empty or He-decorated). When the He-decorated vacancy annihilates with an interstitial (it annihilates because the Frenkel-pair-formation energy is higher than the $\mathrm{He}$ dissociation energy ${ }^{2}$ ), the $\mathrm{He}$ atom is pushed into the interstitial region, where it migrates until it becomes trapped at another vacancy. In this way some monovacancies get decorated with two $\mathrm{He}$ atoms. In stage III, empty vacancies become mobile and annihilate at interstitial clusters or get trapped by other vacancies (with or without $\mathrm{He}$ ). This can cause some divacancies to be decorated with one $\mathrm{He}$ atom. Thus we estimate that the average $[\mathrm{He}] /[V]$ ratio up to the $600-\mathrm{K}$ annealing is close to unity. Above $600 \mathrm{~K}$ the concentration of thermal equilibrium vacancies rapidly exceeds $1 \mathrm{ppm}$ and reduces the $[\mathrm{He}] /[V]$ ratio, since the decorated vacancies are fed by thermal vacancies during the whole annealing time with a constant feeding rate.

The decrease of $\tau_{2}$ between 300 and $400 \mathrm{~K}$ is due to loss of small vacancy clusters created during stage III. The clusters may contain one or two helium atoms, which is not sufficient to keep the clusters stable. The clusters collapse and mainly He-vacancy pairs remain.

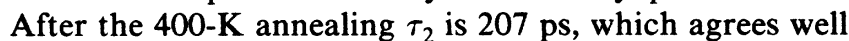
with the theoretical calculations ${ }^{9}$ for the positron trapped at a He-vacancy pair. The lifetime is significantly lower than the lifetime in an empty vacancy (245 ps). This supports the idea that at $400 \mathrm{~K}$ the sample contains monovacancies and divacancies with one or two He atoms.

At $425 \mathrm{~K}, \tau_{2}$ starts to increase. To obtain the longer positron lifetime, three-dimensional vacancy agglomerates have to be formed via migration and clustering of the He-vacancy pairs at this temperature. There are two possible mechanisms for the substitutional $\mathrm{He}$ to migrate and form bubbles. The He-vacancy pair can either dissociate and the $\mathrm{He}$ can migrate interstitially, or the pair can migrate substitutionally by the vacancy mechanism. Helium is deeply trapped by vacancies in aluminum. ${ }^{27,28}$ Thus we conclude that the migration mechanism is most probably substitutional. Then the $\mathrm{He}$ migration energy is ${ }^{2}$

$$
E_{\mathrm{He}}^{M}=E_{V}^{F}+E_{V}^{M}+E_{V}^{T}
$$

where $E_{V}^{F}$ is the vacancy-formation energy and $E_{V}^{M}$ the vacancy-migration energy. $E_{V}^{T}$ is the energy needed to transfer a substitutional $\mathrm{He}$ into a neighboring vacancy, which is expected to be very small. ${ }^{2}$ Thus the migration energy is expected to be nearly equal to the self-diffusion energy.

From the onset temperature of $425 \mathrm{~K}$ we can estimate the migration energy of the He-vacancy pair by using a simple model for the migration. The migration length in the random walk process is $x=\sqrt{6 D t}$, where $t=1800 \mathrm{~s}$ is the annealing time and $D$ is the diffusion constant. From the $\mathrm{He}$ concentration $(1000 \mathrm{ppm})$ we can estimate that the distance between the $\mathrm{He}$ atoms is $\sim 3 \mathrm{~nm}$. Using the Arrhenius relation with a prefactor for self-diffusion in $\mathrm{Al}, 0.05 \mathrm{~cm}^{2} / \mathrm{s},{ }^{29}$ we obtain $E_{\mathrm{He}}^{M}=1.33 \mathrm{eV}$. Due to un- 
TABLE I. Summary of the bubble parameters above $600 \mathrm{~K}$. $T_{\text {ann }}$ is the annealing temperature and $\tau_{2}$ the positron lifetime in the helium bubbles. $n_{\mathrm{He}}$ is the density of helium in the bubbles deduced from Eq. (3). $p$ is the helium pressure from the equation of state (Ref. 3 ) and $r_{B}$ the equilibrium bubble radius.

\begin{tabular}{lcccr}
\hline \hline$T_{\text {ann }}$ & $\tau_{2}$ & $n_{\mathrm{He}}$ & $p$ & $r_{B}$ \\
$(\mathrm{~K})$ & $(\mathrm{ps})$ & (He-atoms/vacancy) & $($ kbar $)$ & $(\AA)$ \\
\hline 600 & 336 & 1.16 & 24.8 & 8 \\
625 & 342 & 1.12 & 23.4 & 9 \\
650 & 354 & 1.04 & 20.1 & 10 \\
675 & 377 & 0.87 & 14.3 & 14 \\
700 & 402 & 0.70 & 9.4 & 21 \\
725 & 410 & 0.64 & 8.2 & 24 \\
750 & 432 & 0.48 & 5.3 & 38 \\
775 & 442 & 0.41 & 4.2 & 47 \\
800 & 450 & 0.35 & 3.5 & 57 \\
825 & 448 & 0.37 & 3.8 & 53 \\
850 & 460 & 0.28 & 2.7 & 74 \\
875 & 462 & 0.27 & 2.6 & 77 \\
900 & 469 & 0.22 & 2.1 & 97 \\
\hline \hline
\end{tabular}

certainties in the estimates (prefactor, distance between He atoms, onset temperature), we estimate the migration energy to be $1.3 \pm 0.1 \mathrm{eV}$. This value is close to the selfdiffusion energy, which is $1.28 \mathrm{eV}$ in $\mathrm{Al}^{29}$ Schiani and Jung $^{5}$ have studied the migration of He-vacancy pairs in aluminum, employing the thermal-helium-desorption technique, and determined the migration energy to be $1.35 \mathrm{eV}$. They concluded that the migration mechanism is substitutional, in accord with our results.

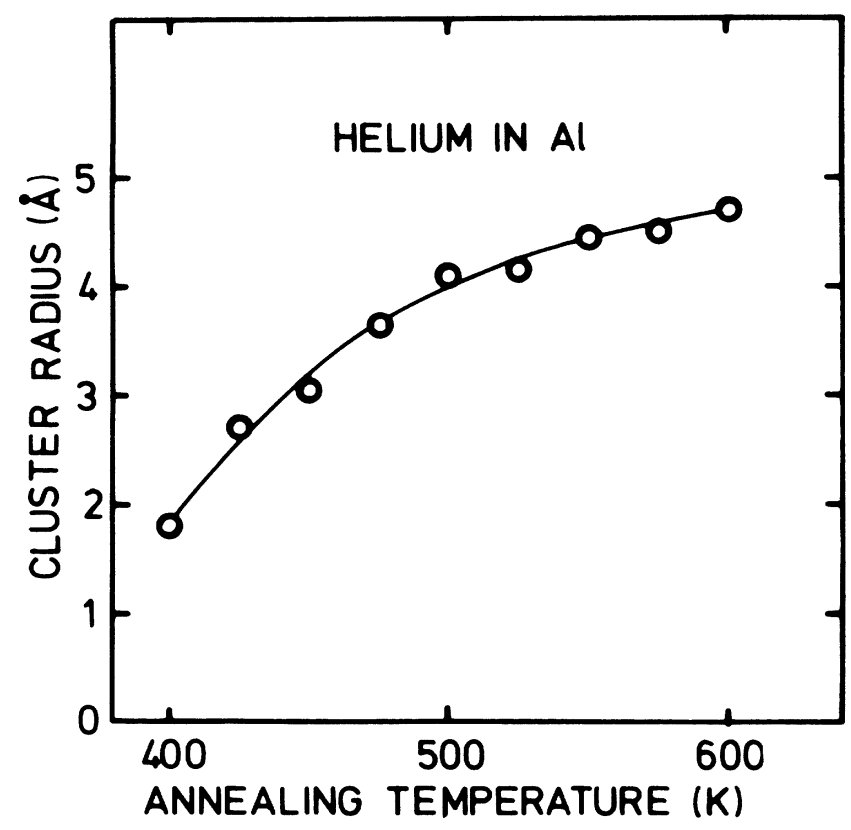

FIG. 3. Vacancy cluster radius vs annealing temperature as determined from the positron-lifetime results. The $[\mathrm{He}] /[\mathrm{V}] \mathrm{ra}-$ tio in the cluster was assumed to be unity and the radius was extracted from the theoretical result (Ref. 13). The line is drawn only to guide the eye. $\tau_{2}$ increases rapidly between 425 and $500 \mathrm{~K}$ because the number of the mobile He-vacancy pairs is high. The mobility of the larger clusters is lower than for single $\mathrm{He}$ vacancy pairs and the increase of $\tau_{2}$ between 500 and 600 $\mathbf{K}$ becomes more slow. The clusters are now growing less effectively. Another possible reason for the slow increase of $\tau_{2}$ above $500 \mathrm{~K}$ is that during the coalescence underpressurized bubbles can eject vacancies (or absorb interstitials from dissociating interstitial loops) in order to reach the thermal equilibrium pressure

$$
p=\frac{2 \gamma}{r_{B}}
$$

where $r_{B}$ is the radius of the spherical cavity and $\gamma$ is the surface tension $\left(=1 \mathrm{~N} / \mathrm{m}\right.$ in aluminum $\left.{ }^{6}\right)$. Then the $[\mathrm{He}] /[V]$ ratio would increase simultaneously with increasing bubble radius. On the other hand, we observe that the $[\mathrm{He}] /[\mathrm{V}]$ ratio after the $600-\mathrm{K}$ annealing is about 1.16 (see Table I) and thus these reactions do not seem to be dominating. Also, when there are 1-50 helium atoms in a cluster, it is doubtful whether the continuum equation of state [and Eq. (3)] can be applied. ${ }^{3}$

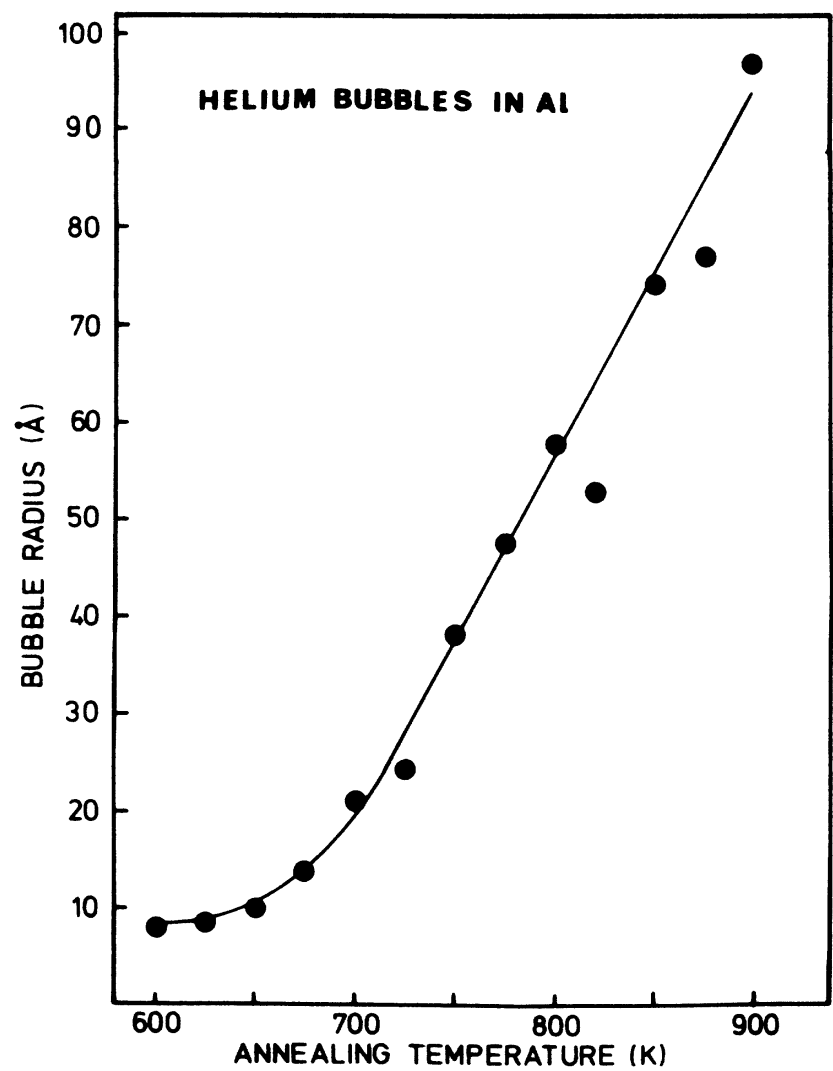

FIG. 4. Helium-bubble radius vs annealing temperature above $600 \mathrm{~K}$. The radius was obtained by adopting the theoretical results by Jensen and Nieminen (Ref. 13) to get the He density and further the equation of state (Ref. 3) to obtain the helium pressure in the bubbles. From the pressure one gets the radius of the bubbles (see Table I). The radius at $900 \mathrm{~K}$ is twice the radius from the TEM micrographs (see Fig. 6). The line is drawn only to guide the eye. 
The lifetime results from 400 to $600 \mathrm{~K}$ are analyzed assuming the $[\mathrm{He}] /[V]$ ratio close to 1 . Then we can use the theoretical result by Jensen and Nieminen ${ }^{13}$ to calculate the size of the He-decorated vacancy clusters from the positron lifetime. The result of the analyses is shown in Fig. 3. The cluster radius (assuming it to be spherical) is seen to increase from $1.8 \AA$ at $400 \mathrm{~K}$ to $4.7 \AA$ at $600 \mathrm{~K}$. The radius of a monovacancy is $1.6 \AA$. The radius must, of course, not be taken too literally, particularly for small clusters, but it rather tells the number of vacancies in the cluster. At $400 \mathrm{~K}$ there are mainly He-monovacancy complexes, while at $600 \mathrm{~K}$ the clusters contain about 25 vacancies and $\mathrm{He}$ atoms.

Since the $[\mathrm{He}] /[V]$ ratio in the He-decorated clusters during (400-600)-K annealings is close to unity (i.e., below the equilibrium pressure for a given radius and density at those temperatures), the pressure-driven growth mechanisms (dislocation-loop punching and Ostwald ripening) can be neglected. The threshold density for loop punching in, e.g., 10- $\AA$ bubbles at room temperature, is $[\mathrm{He}] /[V]=2.11 .^{6}$ Ostwald ripening can be ruled out also due to the strong binding of $\mathrm{He}$ to vacancies in Al. The growth mechanism is thus coalescence via bubble migration.

The step in the lifetime parameters at $600 \mathrm{~K}$ is due to repacking of the sample sandwich. It shows that the packing after the $300-\mathrm{K}$ annealing was not optimal, but the source was slightly off the beam spot. The packing was repeated several times in order to check that it was possible to reproduce the lifetime data measured before repacking. The change in $\tau_{2}$ also shows that the beam spot is not completely homogeneous, but the bubbles in the center of the spot are slightly larger than the bubbles at the edge. This is the reason why the analyses at $600 \mathrm{~K}$ lead to two different cluster radii, $4.7 \AA$ at the edge and around $8 \AA$ in the center, as seen below. Since the analyzing procedures to obtain the radii were different, the effect of the procedure was checked by analyzing the results at $600 \mathrm{~K}$ using both procedures. The resulting radius is 4.7 and $5 \AA$ for the cluster size at the edge of the beam spot. Thus, the resulting difference in the radius at $600 \mathrm{~K}$ is due to the real difference in the radii (and positron lifetime) and not due to the analyzing procedure.

Above $600 \mathrm{~K}$ the rapid increase of $\tau_{2}$ can be assigned to the thermal equilibrium vacancy condensation by the helium-containing clusters (helium bubbles) or the bubble migration and coalescence. The bubbles have reached thermal equilibrium at $600 \mathrm{~K}$. When the vacancy concentration is sufficient, the He pressure in the bubbles can relax to the equilibrium value [given by Eq. (3)] through vacancy absorption. When it is assumed that the bubbles at $600 \mathrm{~K}$ are large $\left(r_{B}>5 \AA\right)$, the size of the cavity does not have an influence on the corresponding positron lifetime. ${ }^{13}$ Then the positron lifetime and the helium density, $n_{\mathrm{He}}$, obey the relation ${ }^{13}$

$$
\tau(\mathrm{ps})=500-23.5 n_{\mathrm{He}}\left(10^{22} \mathrm{~cm}^{-3}\right),
$$

from which the helium density is deduced (cf. Table I). The densities obtained are, in fact, upper limits, since for smaller bubbles $\left(r_{B}<5 \AA\right)$ the size effect would decrease the lifetime ${ }^{13}$ and the density should be lower in order to get the measured lifetime. From the equation of state ${ }^{3}$ the helium pressure is obtained and subsequently the equilibrium bubble radius is deduced from Eq. (3). The bubble radius versus the annealing temperature is presented in Fig. 4. Since the densities are upper limits, the pressures given in Table I are also upper limits and, with the assumption of thermal equilibrium, the bubble radii are lower limits. From Table I one can thus see that the original assumption $\left(r_{B}>5 \AA\right)$ is fulfilled in the whole annealing region above $600 \mathrm{~K}$. Both thermal vacancy condensation and bubble migration-coalescence seem to take place, since both the helium density in the bubbles and the bubble density (correlated to $I_{2}$ ) are decreasing during the annealing.

\section{Temperature dependence of the lifetime spectrum}

After the annealing at $900 \mathrm{~K}$ the temperature dependence of the lifetime parameters between 80 and $340 \mathrm{~K}$ was measured. The results clearly showed that the longer lifetime, $\tau_{2}$, remains constant but its intensity, $I_{2}$, increases with increasing measuring temperature. $\tau_{2}$ was

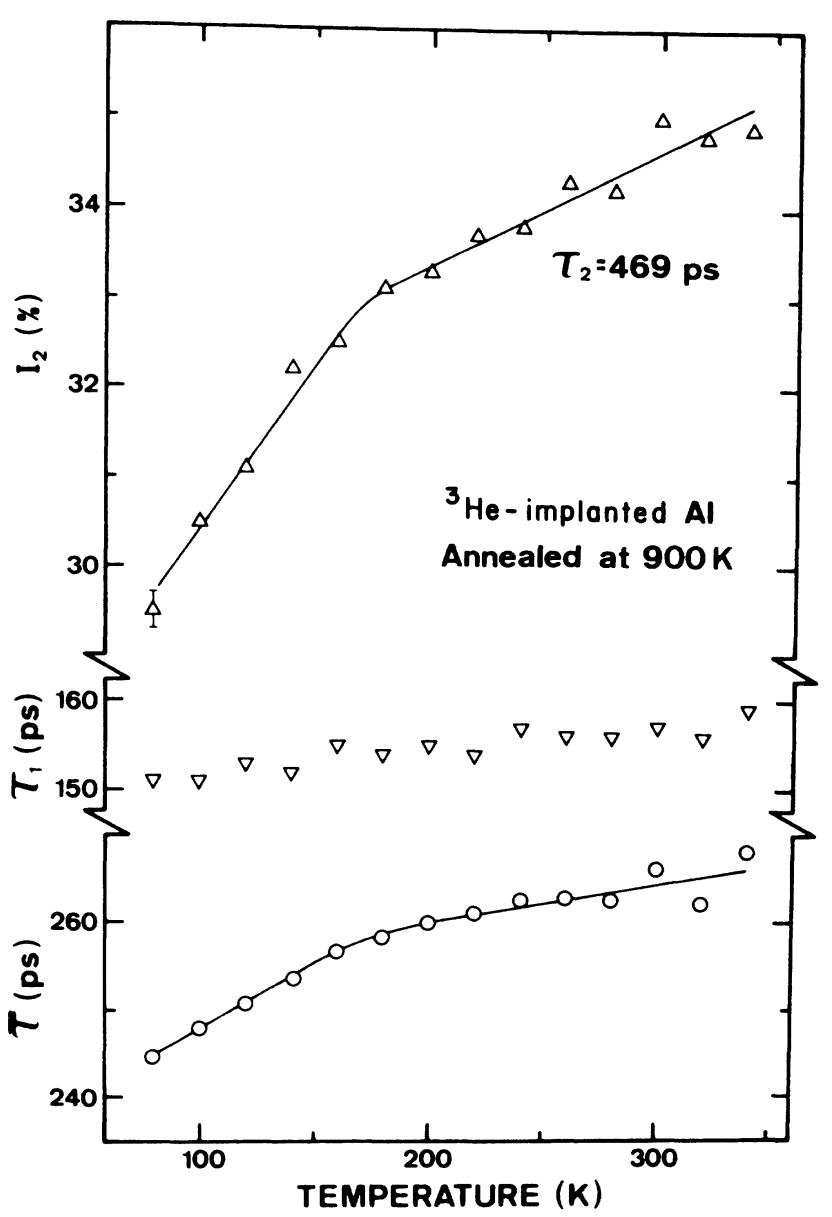

FIG. 5. Temperature dependence of the positron lifetime parameters $\tau_{1}$ and $I_{2}$ between 80 and $340 \mathrm{~K} . \tau_{2}$ is constrained to $469 \mathrm{ps}$ as deduced from a free two-component fit. Also the mean lifetime, $\bar{\tau}=\sum_{i=1}^{N} I_{i} \tau_{i}$, is shown. The lines are drawn only to guide the eye. 
then constrained to $469 \mathrm{ps,}$ the average through the measured temperature range, in order to reduce the statistical scatter. Figure 5 shows the two-exponential fit with $\tau_{2}$ constrained. $I_{2}$ is $29.5 \%$ at $80 \mathrm{~K}$ and increases rapidly, being $33 \%$ at $180 \mathrm{~K}$ and then continues increasing more slowly to $35 \%$ at $340 \mathrm{~K}$.

$\tau_{1}$ in Fig. 5 is increasing with increasing trapping to He bubbles, while the trapping model would suggest opposite behavior. $\tau_{1}$ is an average of the modified "bulk" lifetime in the implanted region (shorter than the positron lifetime in defect-free Al) and the lifetime in the defect-free nonimplanted region. The fraction of the modified "bulk" lifetime in $\tau_{1}$ is decreasing with increasing temperature due to stronger trapping, and thus $\tau_{1}$ is increasing.

The temperature dependence after $900-\mathrm{K}$ annealing is very similar to the results of Nieminen et al. ${ }^{30}$ and Linderoth et al., ${ }^{31}$ who studied neutron-irradiated aluminum containing large voids. Since our samples were annealed at $900 \mathrm{~K}$, all competing trapping centers are expected to have annealed out, ${ }^{32}$ and we conclude that the specific trapping rate to large helium bubbles is temperature dependent.

\section{E. TEM results after $\mathbf{9 0 0 - K}$ annealing}

One of the specimens was investigated with TEM after 900-K annealing. The bubbles are found to be faceted

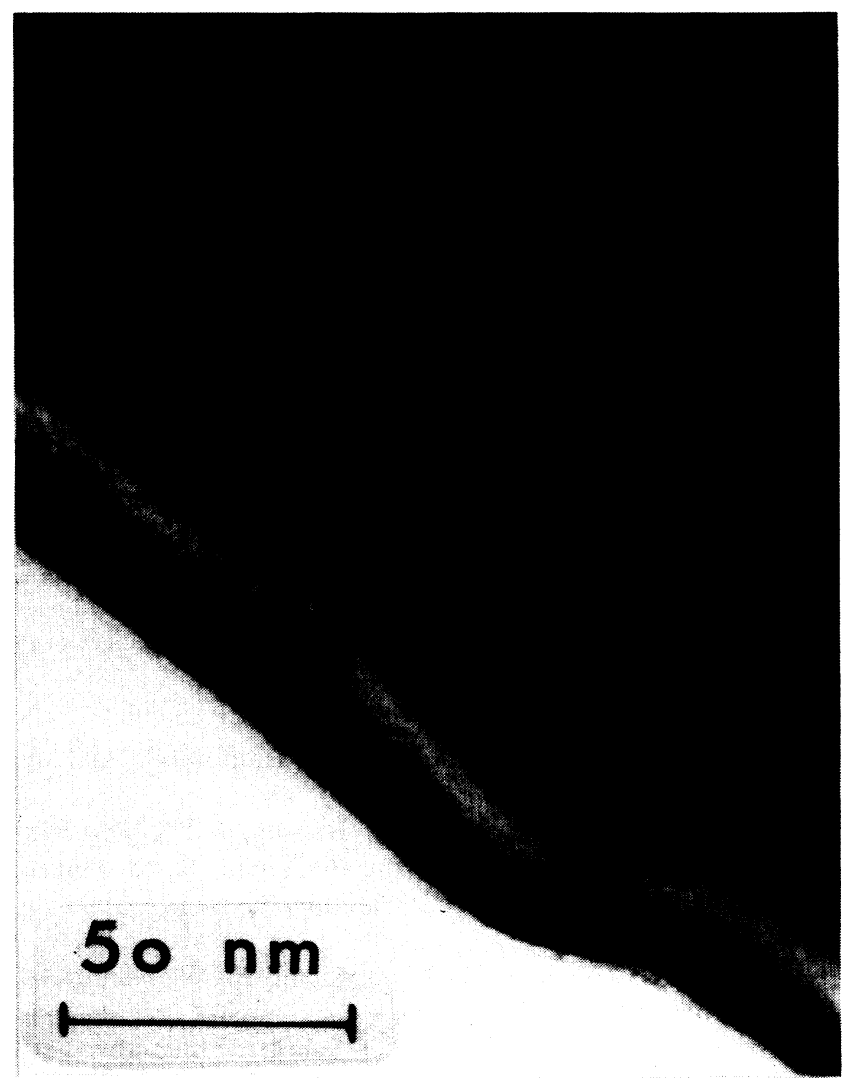

FIG. 6. TEM micrograph showing the polygon-shaped bubbles as dark spots. The surrounding bright ring is due to overfocusing. The thickness fringes are caused by excitation of the (111) reflection. The size distribution is shown in Fig. 7. Incident beam, (123); $H V, 300 \mathrm{kV}$; defocusing, $+1.1 \mu \mathrm{m}$. polygons and not spherical, as seen in Fig. 6. The distribution of the bubble radii is given in Fig. 7 and is based on the observation of 100 bubbles. The distribution gives a mean radius of $45 \AA$. The bubble concentration was determined in two different areas of the sample to be $2.8 \times 10^{15}$ and $3.0 \times 10^{15} \mathrm{~cm}^{-3}$, respectively. Thus the bubble concentration is $(2.9 \pm 0.8) \times 10^{15} \mathrm{~cm}^{-3}$, where the uncertainty is due to statistics ( 44 bubbles), area determination, and thickness determination.

The mean bubble radius observed by TEM (45 $\AA$ ) is significantly smaller than the radius deduced from the positron-annihilation data $(97 \AA)$. The discrepancy can partly be explained by the fact that the bubbles are not spherical, which was one of the assumptions in the calculations. The system tends to minimize the total energy and thus the shape indicates that some crystal faces have lower surface tension than the others. The surface tension in Eq. (3) will thus contain large errors. With the distribution of bubble sizes the positron-lifetime technique tends to overestimate the mean bubble size, because large bubbles have the largest trapping rate. From Eq. (4) one can see that the important factor is the difference between the empty-void value [500 ps, cf. Eq. (4)] and the measured positron lifetime. Thus a small uncertainty of the measured positron lifetime leads to large uncertainties in the deduced bubble radius. This is particularly true for large bubbles with a low helium density because then the positron lifetime becomes close to the empty-

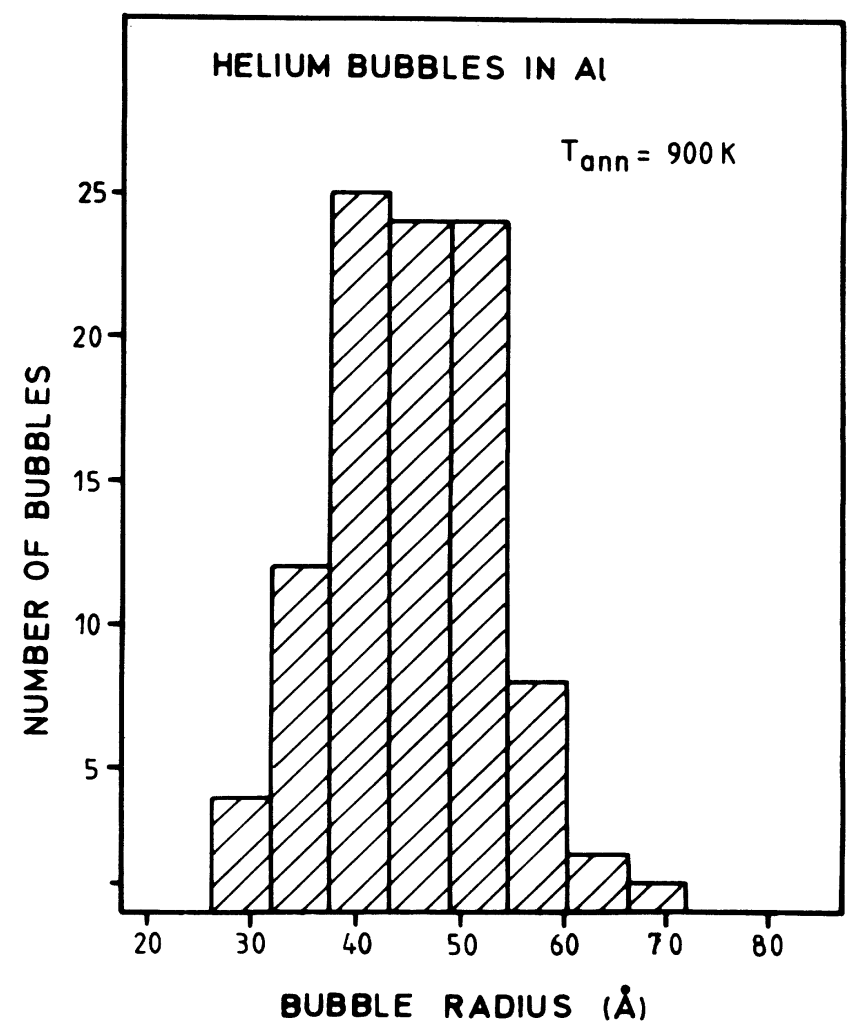

FIG. 7. Size distribution of the bubbles obtained from the TEM micrographs (see Fig. 6). The total number of bubbles $(100)$ is collected from two different areas of the sample. 
void value. Close to the positron lifetime of 450 ps an uncertainty of $4 \%$ thus leads to an uncertainty of $40 \%$ in the determined bubble radius. A deviation from the employed empty-void value will also bring uncertainties to the bubble-parameter deductions (e.g., the positron lifetime at an Al surface has been measured to be $580 \mathrm{ps},{ }^{33}$ which should be equal to the positron lifetime in large voids, i.e., at internal surfaces). Combined TEM and positron results have raised questions about the positron lifetime in voids and/or the surface tension in helium bubbles.

\section{SUMMARY}

The positron-lifetime technique has been applied to study the formation and growth of helium bubbles in aluminum. The early nucleation stage of the bubbles is observed during the isochronal annealing. Helium is seen to migrate substitutionally with an activation energy of $1.3 \pm 0.1 \mathrm{eV}$ and agglomerate into helium-filled clusters around $450 \mathrm{~K}$. In addition, the annealing stages I-III are observed. The positron lifetime is linked to the helium density inside the bubbles. The resulting growth mechanism is consistently explained by bubble migration or coalescence and thermal-equilibrium-vacancy condensation. This experiment also emphasizes the unique role of the positron-annihilation technique to study defectimpurity reactions and agglomeration, particularly in the submicroscopic regime.

\section{ACKNOWLEDGMENTS}

This research has been supported in part by the Nordic Committee for Accelerator-Based Research (NOAC).
*Present address: Medimatic, Gersonsvej 9, DK-2900 Hellerup, Denmark.

†Present address: Laboratory of Physics, Helsinki University of Technology, SF-02150 Espoo, Finland.

${ }^{1}$ Proceedings of the International Symposium on Fundamental Aspects of Helium in Metals, edited by $\mathbf{H}$. Ullmaier, [Radiat. Effects 78, 1 (1983)].

${ }^{2}$ H. Ullmaier, Nucl. Fusion 24, 1039 (1984).

${ }^{3} \mathrm{H}$. Trinkaus, in Proceedings of the International Symposium on Fundamental Aspects of Helium in Metals, Ref. 1, p. 189.

${ }^{4} \mathrm{G}$. J. Thomas, in Proceedings of the International Symposium on Fundamental Aspects of Helium in Metals, Ref. 1, p. 37.

${ }^{5} \mathrm{~V}$. Sciani and P. Jung, in Proceedings of the International Symposium on Fundamental Aspects of Helium in Metals, Ref. 1, p. 87.

${ }^{6}$ S. E. Donnelly, Radiat. Effects 90, 1 (1985).

${ }^{7}$ Positrons in Solids, Vol. 12 of Topics in Current Physics, edited by P. Hautojärvi (Springer, Berlin, 1979).

${ }^{8}$ P. Hautojärvi, J. Heiniö, M. Manninen, and R. Nieminen, Philos. Mag. 35, 973 (1977).

${ }^{9}$ H. E. Hansen, R. M. Nieminen, and M. J. Puska, J. Phys. F 14, 1299 (1984).

${ }^{10}$ C. L. Snead, A. N. Goland, and F. W. Wiffen, J. Nucl. Mater. 64, 195 (1977).

${ }^{11}$ H. E. Hansen, H. Rajainmäki, R. Talja, M. D. Bentzon, R. M. Nieminen, and K. Petersen, J. Phys. F 15, 1 (1985).

${ }^{12}$ K. O. Jensen, M. Eldrup, B. N. Singh, and M. Victoria, J. Phys. F (to be published).

${ }^{13}$ K. O. Jensen and R. M. Nieminen, Phys. Rev. B 36, 8219 (1987).

${ }^{14}$ H. E. Hansen, R. Talja, H. Rajainmäki, H. K. Nielsen, B. Nielsen, and R. M. Nieminen, Appl. Phys. A 36, 81 (1985).

${ }^{15}$ S. Linderoth, H. Rajainmäki, and R. M. Nieminen, Phys. Rev. B 35, 5524 (1987).

${ }^{16} \mathrm{H}$. Bethe, in Handbuch der Physik, edited by H. Geiger and K. Scheel (Springer, Berlin, 1933), vol. 24, p. 491.

${ }^{17}$ N. I. Venikov, and N. I. Chumakov, At. Energiya 17, 503 (1964) [Sov. Atom. Energ. 17, 1269 (1964)].
${ }^{18}$ S. Linderoth, H. E. Hansen, B. Nielsen, and K. Petersen, Appl. Phys. A 33, 25 (1984).

${ }^{19}$ I. K. MacKenzie, in Positron Solid-State Physics, edited by W. Brandt and A. Dupasquier (North-Holland, Amsterdam, 1983).

${ }^{20}$ P. Kirkegaard, M. Eldrup, O. E. Mogensen, and N. J. Pedersen, Comput. Phys. Commun. 23, 307 (1981).

${ }^{21}$ See, e.g., J. Gittus, Irradiation Effects in Crystalline Solids (Applied Science, London, 1978).

${ }^{22}$ H. Ullmaier and W. Schilling, in Physics of Modern Materials, Vol. I (IAEA, Vienna, 1980), p. 301.

${ }^{23}$ H. Rajainmäki, S. Linderoth, and R. M. Nieminen, in Vacancies and Interstitials in Metals and Alloys, edited by $\mathrm{C}$. Abromeit and H. Wollenberger (Trans Tech, Switzerland, 1987), p. 611.

${ }^{24}$ W. Schilling, G. Burger, K. Isebeck, and H. Wenzl, in Vacancies and Interstitials in Metals, edited by A. Seeger, D. Schumacher, W. Schilling, and J. Diehl (North-Holland, Amsterdam, 1969), p. 255.

${ }^{25} \mathrm{H}$. Rajainmäki and S. Linderoth (unpublished).

${ }^{26}$ R. M. Nieminen and J. Laakkonen, Appl. Phys. 20, 181 (1979).

${ }^{27}$ W. Schilling, in Point Defects and Defect Interactions in Metals, edited by J. Takamura, M. Doyama, and M. Kiritani (University of Tokyo Press, Tokyo, 1982), p. 303.

${ }^{28}$ M. J. Puska and R. M. Nieminen, Phys. Rev. B 29, 5382 (1984).

${ }^{29}$ N. L. Peterson, J. Nucl. Mater. 69/70, 3 (1978).

${ }^{30}$ R. M. Nieminen, J. Laakkonen, P. Hautojärvi, and A. Vehanen, Phys. Rev. B 19, 1397 (1979).

${ }^{31}$ S. Linderoth, M. D. Bentzon, H. E. Hansen, and K. Petersen, in Positron Annihilation, edited by P. C. Jain, R. M. Singru, and K. P. Gopinathan (World Scientific, Singapore, 1985), p. 494.

${ }^{32}$ K. Petersen, N. Thrane, G. Trumpy, and R. W. Hendricks, Appl. Phys. 10, 85 (1976).

${ }^{33}$ K. G. Lynn, W. E. Frieze, and P. J. Schultz, Phys. Rev. Lett. 52, 1137 (1984). 


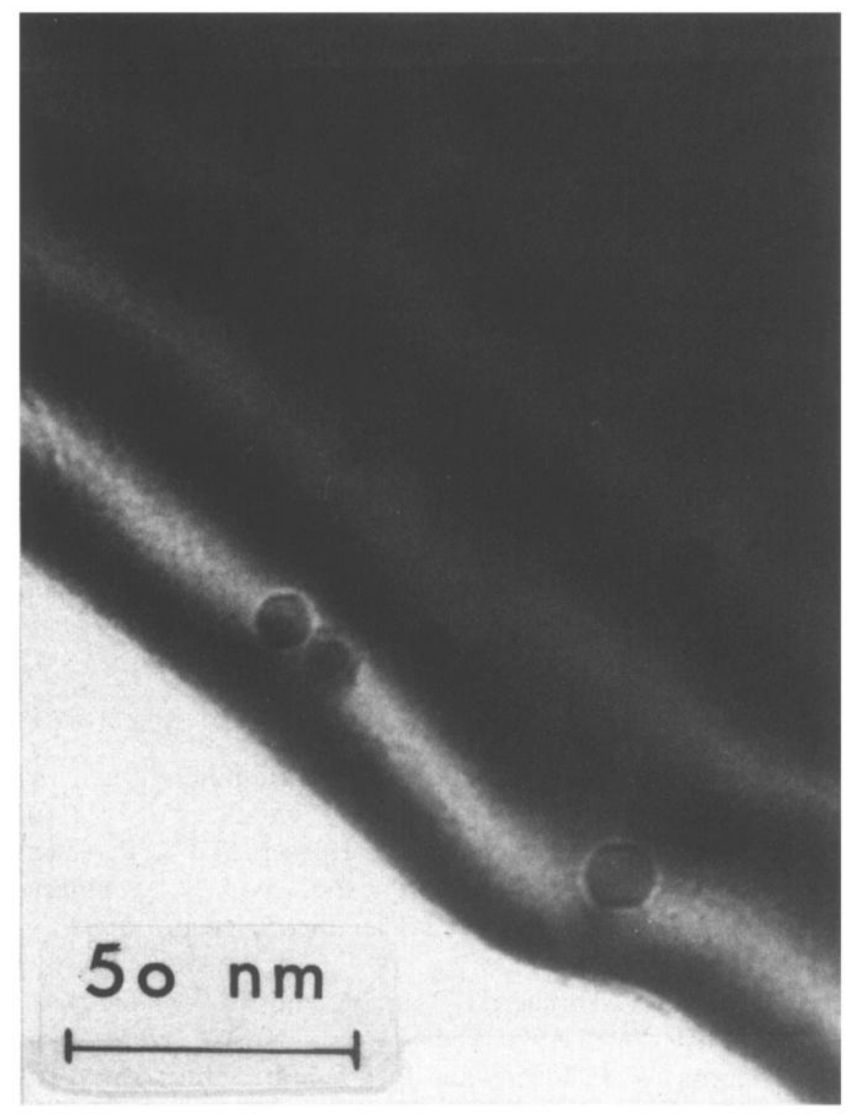

FIG. 6. TEM micrograph showing the polygon-shaped bubbles as dark spots. The surrounding bright ring is due to overfocusing. The thickness fringes are caused by excitation of the (111) reflection. The size distribution is shown in Fig. 7. Incident beam, (123); $H V, 300 \mathrm{kV}$; defocusing, $+1.1 \mu \mathrm{m}$. 